\title{
On Gray Images of Constacyclic Codes
}

Mustafa Özkan, ${ }^{1, *}$,Figen Öke

${ }^{1}$ Department of Mathematics, University of Trakya, Edirne, Turkey

${ }^{2}$ Department of Mathematics, University of Trakya, Edirne, Turkey

Abstract This paper deals with constacyclic codes over a new ring. Gray images of these codes over those rings are obtained. The relations between these codes and their Gray images are given.

\section{Introduction}

Cyclic and constacyclic codes over finite rings with two variables were investigated in many different studies. Transformations preserving distances from these rings to another finite ring or finite fields are studied. Construction of $(1+v)$-contacyclic codes over the ring was studied in [6] and Gray images of these codes are given. Cyclic codes, quasi-cyclic codes over $\mathbb{F}_{2}+u \mathbb{F}_{2}+v \mathbb{F}_{2}+u v \mathbb{F}_{2}$ and their dual were studied by different authors $[3,6,7]$.

Using these studies, under the conditions $u^{2}=v^{2}=u v=0,(1+v)$ constacyclic codes over $\mathbb{F}_{2}+u \mathbb{F}_{2}+v \mathbb{F}_{2}$ were investigated in [8].

Under the conditions $u^{2}=v^{3}=u v=0$, the authors have shown in [1] the Gray images of the $(1+v)$-constacyclic codes over $\mathbb{F}_{2}+v \mathbb{F}_{2}+u \mathbb{F}_{2}+v^{2} \mathbb{F}_{2}$ are quasi-cyclic codes over the ring $R=\mathbb{F}_{2}+v \mathbb{F}_{2}+v^{2} \mathbb{F}_{2}$.

\footnotetext{
* Corresponding author: mustafaozkan@trakya.edu.tr
} 


\section{Preliminaries}

Throughout this paper $R_{u, v}:=\mathbb{F}_{2}+v \mathbb{F}_{2}+u \mathbb{F}_{2}+u^{2} \mathbb{F}_{2}$ and $R_{v}:=\mathbb{F}_{2}+v \mathbb{F}_{2}$ under the conditions $u^{3}=v^{2}=u v=0$ are two commutative local rings. Obviously $R_{v}$ is a subring of $R_{u, v}$ whose $R_{v}$ is a finite chain ring and $R_{u, v}$ is a finite non-chain ring. Moreover $R_{u, v}$ and $R_{v}$ are vector spaces over $\mathbb{F}_{2}$ with basis $\left\{1 ; v ; u ; u^{2}\right\}$ and $\{1 ; v\}$, respectively.

The submodules of $\left(R_{u, v}\right)^{n}$ (resp. $\left.\left(R_{v}\right)^{n}\right)$ are called linear codes of length $n$ over $R_{u, v}$ (resp. $R_{v}$ ).

Definition: The Hamming weight over $\mathbb{F}_{2}$ is defined as;

$$
w_{H}(m)= \begin{cases}0, & m=0 \\ 1, & m=1\end{cases}
$$

Hamming weight of a code-word with length $n, m=\left(m_{0}, \ldots, m_{n-1}\right) \in \mathbb{F}_{2}^{n}$ is of the form $w_{H}(m)=\sum_{i=0}^{n-1} w_{H}\left(m_{i}\right)$.

The Lee weight $\quad w_{L}: R_{v} \rightarrow \mathbb{N}$ is defined by letting $w_{L}(0)=0$, $w_{L}(1)=w_{L}(1+v)=1, w_{L}(v)=2$.

Then $\quad w_{L}(r)=\sum_{i=1}^{n-1} w_{L}\left(r_{i}\right) \quad$ is satisfied for each element $r=\left(r_{0}, \ldots, r_{n-1}\right) \in R_{v}^{n}$.

Definition: The special weight over the ring $R_{u, v}$ as;

$$
w_{\text {Special }}(k):= \begin{cases}0 & ; k=0 \\ 2 & ; k=1+u, 1+v+u \\ 6 & ; k=u, 1,1+u^{2}, u+u^{2}, 1+v+u+u^{2} \\ 4 & ; \text { otherwise }\end{cases}
$$


So the special weight of a codeword $k=\left(k_{0}, \ldots, k_{n-1}\right) \in R_{u, v}^{n}$ is of the form;

$$
w_{s}(k)=\sum_{i=0}^{n-1} w_{s}\left(k_{i}\right)
$$

Definition: The $F_{2}$ - linear map $\Phi: R_{u, v}^{n} \rightarrow R_{v}^{4 n}$ is given by: $\Phi(1)=e_{1}+v e_{4}$, $\Phi(v)=e_{2}+v e_{4}, \quad \Phi(u)=e_{3}+v e_{4}$, and $\Phi\left(u^{2}\right)=e_{4}$, where $e_{1}:=(0,1,0,1)$, $e_{2}:=(0,1,1,0), \quad e_{3}:=(0,0,1,1), \quad e_{4}:=(1,1,1,1) . \quad$ The map $\Phi: R_{u, v}^{n} \rightarrow R_{v}^{4 n}$ extends to $\left(R_{u, v}\right)^{n}$ as follows :

$\Phi\left(c_{1}, \ldots, c_{n}\right)=\left(\Phi\left(c_{1}\right), \ldots, \Phi\left(c_{n}\right)\right)$ for any $\left(c_{1}, \ldots, c_{n}\right) \in\left(R_{u, v}\right)^{n}$.

\section{Constacyclic, Cyclic and Quasi-cyclic codes}

A cyclic shift on $R_{u, v}^{n}$ is a permutation $\sigma$ such that

$$
\sigma\left(c_{o}, c_{1}, \ldots, c_{n-1}\right)=\left(c_{n-1}, c_{0}, \ldots, c_{n-2}\right) .
$$

A linear code $C$ over $R_{u, v}$ of length $n$ is said to be cyclic code if it is satisfied the equality $\sigma(C)=C$.

A $\left(1+u^{2}\right)$ - constacyclic shift $\gamma$ acts on $R_{u, v}^{n}$ as

$$
\gamma\left(c_{o}, c_{1}, \ldots, c_{n-1}\right)=\left(\left(1+u^{2}\right) \cdot c_{n-1}, c_{0}, \ldots, c_{n-2}\right)
$$

A linear code $C$ over $R_{u, v}$ of length $n$ is said to be $\left(1+u^{2}\right)$-constacyclic code if it is satisfied the equality $\gamma(C)=C$.

A cyclic shift on $R_{v}^{4 n}$ is a permutation $\tau$ such that

$$
\tau\left(d_{o}, d_{1}, \ldots, d_{4 n-1}\right)=\left(d_{4 n-1}, d_{0}, \ldots, d_{4 n-2}\right) .
$$


Let $D \subseteq R_{v}^{4 n}$ is a linear code. If $\tau(D)=D$ then $D$ is called a cyclic code over $R_{v}$.

A cyclic shift on $R_{v}^{4 n}$ is a permutation $\tau^{\otimes 2}$ such that $\tau^{\otimes 2}\left(c_{o}, c_{1}, \ldots, c_{4 n-1}\right)=\left(c_{2 n-1}, c_{0}, \ldots, c_{2 n-2}, c_{4 n-1}, c_{2 n}, \ldots, c_{4 n-2}\right)$.

If $\tau^{\otimes 2}(D)=D$ then $D$ is called a quasicyclic code of index 2 over $R_{v}$.

We have for $i \in\{1 ; 2 ; 3\}, \tau^{\otimes 2}\left(e_{i}\right)=e_{i}$ and $\sigma\left(e_{4}\right)=e_{4}$.

A $(1+v)-$ constacyclic shift on $R_{v}^{4 n}$ is a permutation $\beta$ such that

$$
\beta\left(d_{o}, d_{1}, \ldots, d_{4 n-1}\right)=\left((1+v) \cdot d_{4 n-1}, d_{0}, \ldots, d_{4 n-2}\right) .
$$

Let $D \subseteq R_{v}^{4 n}$ is a linear code. If $\beta(D)=D$ then $D$ is called a $(1+v)$-constacyclic code over $R_{v}$.

\section{Main Results}

Lemma 4.1 : The equality $\Phi . \gamma=\sigma^{\otimes 2} . \Phi$ is satisfied.

Proof : $\gamma(k)=\gamma\left(k_{0}, \ldots, k_{n-1}\right)=\left(\left(1+u^{2}\right) k_{n-1}, k_{0}, \ldots, k_{n-2}\right)$

is satisfied for each $k=\left(k_{0}, \ldots, k_{n-1}\right) \in R_{u, v}^{n}$, where $k_{i}=r_{i}+s_{i} u+t_{i} u^{2}$, $0 \leq i \leq n-1$.

Then it is obtained;

$$
\begin{aligned}
\Phi(\gamma(k))= & \Phi\left(r_{n-1}+s_{n-1} u+\left(r_{n-1}+t_{n-1}\right) u^{2}, r_{0}+s_{0} u+t_{0} u^{2}, \ldots, r_{n-2}+s_{n-2} u+t_{n-2} u^{2}\right) \\
= & \left(r_{n-1}+t_{n-1}, t_{0}, t_{1}, \ldots, t_{n-2}, t_{n-1}, r_{0}+t_{0}, \ldots, r_{n-2}+t_{n-2}, r_{n-1}+s_{n-1}+t_{n-1}, s_{0}+t_{0}, \ldots, s_{n-1}+t_{n-1},\right. \\
& \left.r_{0}+s_{0}+t_{0}, \ldots, r_{n-2}+s_{n-2}+t_{n-2}\right)
\end{aligned}
$$

On the other hand, 


$$
\begin{aligned}
\Phi(k)= & \Phi\left(k_{0}, k_{1}, \ldots, k_{n-1}\right)=\Phi\left(r_{0}+s_{0} u+t_{0} u^{2}, r_{1}+s_{1} u+t_{1} u^{2}, \ldots, r_{n-1}+s_{n-1} u+t_{n-1} u^{2}\right) \\
= & \left(t_{0}, t_{1}, \ldots, t_{n-1}, r_{0}+t_{0}, r_{1}+t_{1}, \ldots, r_{n-1}+t_{n-1}, s_{0}+t_{0}, s_{1}+t_{1}, \ldots, s_{n-1}+t_{n-1},\right. \\
& \left.r_{0}+s_{0}+t_{0}, r_{1}+s_{1}+t_{1}, \ldots, r_{n-1}+s_{n-1}+t_{n-1}\right)
\end{aligned}
$$

Then it is seen that

$$
\begin{aligned}
& \sigma^{\otimes 2}(\Phi(c))= \\
& \sigma^{\otimes 2}\left(t_{0}, t_{1}, \ldots, t_{n-1}, r_{0}+t_{0}, r_{1}+t_{1}, \ldots, r_{n-1}+t_{n-1}, s_{0}+t_{0}, s_{1}+t_{1}, \ldots, s_{n-1}+t_{n-1},\right. \\
& \left.r_{0}+s_{0}+t_{0}, r_{1}+s_{1}+t_{1}, \ldots, r_{n-1}+s_{n-1}+t_{n-1}\right) \\
& =\left(r_{n-1}+t_{n-1}, t_{0}, t_{1}, \ldots, t_{n-2}, t_{n-1}, r_{0}+t_{0}, \ldots, r_{n-2}+t_{n-2}, r_{n-1}+s_{n-1}+t_{n-1}, s_{0}+t_{0}, \ldots, s_{n-1},\right. \\
& \left.r_{0}+s_{0}+t_{0}, \ldots, r_{n-2}+s_{n-2}+t_{n-2}\right) \\
& =\Phi(\gamma(k))
\end{aligned}
$$

Theorem 4.2.: $C$ is a $\left(1+u^{2}\right)$-constacyclic code over $R_{u, v}$ with length $n$ if and only if $\Phi(C)$ is a quasi-cyclic code of second order over $R_{v}$ with length $4 n$.

Proof: If $C$ is a $\left(1+u^{2}\right)$-constacyclic code with $\mathrm{n}$ length over the ring $R_{u, v}$, $\gamma(C)=C$. Then $\Phi(\gamma(C))=\Phi(C)$

Using the equality from the previous lemma the equality is obtained:

$$
\sigma^{\otimes 2}(\Phi(\gamma(C))=\Phi(\gamma(C))=\Phi(C) .
$$

Then we have the code $\Phi(C)$ is a quasi-cyclic code of length 4 with second order.

Conversely; if $\Phi(C)$ is a quasi-cyclic code over $R_{v}$ with length $4 \mathrm{n}$ and of second order, $\sigma^{\otimes 2}(\Phi(C))=\Phi(C)$ is satisfied.

Using the previous lemma the equality $\Phi(\gamma(C))=\sigma^{\otimes 2}(\Phi(C))=\Phi(C)$ is obtained. 
Since $\Phi$ is injective, using the above equality; it is seen that $\gamma(C)=C$. It means that $C$ is a $\left(1+u^{2}\right)$-constacyclic code with length $n$ over the ring $R_{u, v}$.

\section{Conclusion}

In this paper, $\left(1+u^{2}\right)$ - constacyclic codes over a ring isomorphic to $R_{u, v}$ are studied, and a new weight function on $R_{u, v}$ is defined.

\section{References}

1. P. Udomkavanich, S. Jitman, Int.J.Contemp. Math. Sciences, 4(26), 1265-1272, (2009)

2. W. C. Huffman, V. Pless, Cambridge, (2003)

3. M. Ozkan, F. Oke, AIP Conf. Proc., 1926, 020034-1-020034-3, (2018)

4. S. Roman, Graduate Texts in Mathematics, Springer Verlag, (1992)

5. M. Özkan, F.Öke, Mathematical Sciences and Applications E-Notes, 4(1), 40-44, (2016)

6. M. Özkan, F. Öke, Palestine Journal of Mathematics, 6(2), 241-245, (2017)

7. J.F.Qian, L.N.Zhang, S.X Zhu, Applied Mathematics Letters, 19, 820$823,(2006)$

8. Xu Xioafang, Computer Engineering and Applications, 49(12),77-79, (2013) 